\title{
Epidermal growth factor induces cytokeratin 19 expression accompanied by increased growth abilities in human hepatocellular carcinoma
}

\author{
Norihide Yoneda ${ }^{1,2}$, Yasunori Sato ${ }^{1}$, Azusa Kitao ${ }^{1,2}$, Hiroko Ikeda ${ }^{3}$, Seiko Sawada-Kitamura ${ }^{3}$, Masami Miyakoshi ${ }^{1}$, \\ Kenichi Harada', Motoko Sasaki ${ }^{1}$, Osamu Matsui ${ }^{2}$ and Yasuni Nakanuma ${ }^{1}$
}

Cytokeratin (CK) 19-positive hepatocellular carcinoma (HCC) has been reported to have a poor prognosis. The mechanism of the development of CK19-positive HCC remains to be studied. To clarify this, in vitro experiments were performed using human HCC cell lines (PLC-5, HepG2), and the phenotypic changes after stimulation with several growth factors were examined using quantitative reverse transcriptase $P C R$, western blotting, and immunofluorescence staining. In vivo experiments using human HCC specimens obtained from a total of 78 patients and clinicopathological analysis were also performed. Among the growth factors tested, epidermal growth factor (EGF) had prominent effects on inducing CK19 expression in PLC- 5 and HepG2, which was accompanied by the reduced expression of $\alpha$-fetoprotein in PLC-5. The induction of CK19 expression after EGF stimulation was accompanied by the phosphorylation of c-Jun-N-terminal kinase (JNK)/stress-activated protein kinase, which was blocked by the addition of JNK inhibitors. EGF also increased proliferative abilities and invasive properties of the HCC cell lines. In vivo, 9 (12\%) of $78 \mathrm{HCC}$ cases showed positive immunohistochemical staining of CK19. The extent of positive immunhistochemical signals of EGF, EGF receptor (EGFR), and JNK expression was significantly intense in CK-19-positive HCC than those of CK19-negative HCC. Clinicopathological analysis showed that CK19-positive HCC had a high incidence of portal vein invasion, extrahepatic metastasis and an early relapse, which was associated with the worsened 2-year disease free survival. These results indicate that the activation of the EGF-EGFR signaling pathway is associated with the development of CK19-positive HCC, and the EGF-induced increase in growth abilities of HCC may account for the poor prognosis of the patients.

Laboratory Investigation (2011) 91, 262-272; doi:10.1038/labinvest.2010.161; published online 20 September 2010

KEYWORDS: CK19-positive HCC; EGF; EGFR; histogenesis; prognosis

Hepatocellular carcinoma (HCC) is the fifth most important cancer worldwide, and is the third most common cause of death from cancer. ${ }^{1}$ Its incidence and mortality rates have increased in recent years. ${ }^{2}$ The high rate of recurrence or metastases after curative resection leads to worse prognosis in HCC. ${ }^{3,4}$

Recently, several authors have reported the positive immunoexpression of cytokeratin (CK) 19 in HCC, and CK19positive HCC has been shown to have a high metastatic potential, which is associated with a poor prognosis. ${ }^{5-10}$ The expression of CK19 in HCC is implicated as a novel predictor for the poor prognosis of the patients after curative resection. ${ }^{8,11,12}$ Although the clinical significance of CK19-positive
HCC seems to be establishing, the mechanism underlying the development of CK19-positive HCC remains to be unclear.

$\mathrm{CK}$ is cytoskeletal intermediate filaments, and different epithelial cells express characteristic combinations of $\mathrm{CK}$ polypeptides depending on the organ or on the type of differentiation. ${ }^{13,14}$ In normal liver, hepatocytes express CK8 and CK18, and cholangiocytes express CK7 and CK19. ${ }^{15,16}$ Bipotential hepatic progenitor cells have been shown to express CK7 and CK19. ${ }^{17}$

Previous studies showed that rat hepatocytes had a potential to differentiate into bile duct-like cells in vitro. ${ }^{18-22}$ In these studies, epidermal growth factor (EGF) and hepatocytes growth factor (HGF) are potent inducers of biliary

\footnotetext{
${ }^{1}$ Department of Human Pathology, Kanazawa University Graduate School of Medicine, Kanazawa, Japan; ${ }^{2}$ Department of Radiology, Kanazawa University Graduate School of Medicine, Kanazawa, Japan and ${ }^{3}$ Division of Diagnostic Pathology, Kanazawa University Hospital, Kanazawa, Japan Correspondence: Dr Y Nakanuma, MD, PhD, Department of Human Pathology, Kanazawa University Graduate School of Medicine, 13-1 Takara-machi, Kanazawa 9208640, Japan. E-mail: pbcpsc@kenroku.kanazawa-u.ac.jp 
phenotype in rat hepatocytes. ${ }^{18-22}$ Transforming growth factor- $\beta 1$ (TGF- $\beta 1$ ) and basic fibroblast growth factor (FGF-b) are also well-known growth factors that have key roles in the development and differentiation of the liver. ${ }^{23-26}$ These observations suggest that the expression of CK19, one of the characters of biliary CK, may be induced in HCC after stimuli by a certain kind of growth factors, accounting for the development of CK19-positive HCC.

Understanding the developmental mechanism of CK19positive HCC is particularly important, because it may lead to a novel therapeutic approach for the carcinoma with a poor prognosis. To clarify the histogenesis of CK19-positive HCC, this study was conducted using human HCC cell lines, and in vivo experiments using human HCC specimens and clinicopathological analysis were also performed.

\section{MATERIALS AND METHODS Cell Culture}

Human HCC cell lines (PLC-5, HepG2) were purchased from the Health Science Research Resources Bank (Osaka, Japan), and were maintained with Dulbecco modified Eagle medium supplemented with $10 \%$ fetal bovine serum (FBS) and $1 \%$ antibiotics-antimycotic (all from Invitrogen, Carlsbad, CA, USA). Cells were incubated in $7.5 \% \mathrm{CO}_{2}$ at $37^{\circ} \mathrm{C}$. The cells were treated with EGF $(30 \mathrm{ng} / \mathrm{ml})$, TGF- $\beta 1(10 \mathrm{ng} / \mathrm{ml})$, HGF $(50 \mathrm{ng} / \mathrm{ml})$, and FGF-b $(50 \mathrm{ng} / \mathrm{ml})$ for 5 days (all from R\&D systems, Minneapolis, MN, USA). Treatment with growth factors was carried out with culture medium that did not contain FBS. To block the activity of c-Jun-N-terminal kinase (JNK), JNK inhibitor I ( $1 \mu \mathrm{M}$; Merck, Darmstadt, Germany) and JNK inhibitor II ( $50 \mathrm{nM}$; Merck) were used.

\section{Reverse transcriptase PCR (RT-PCR) and Quantitative Real-Time PCR}

RT-PCR was performed using total RNA $(1 \mu \mathrm{g})$ extracted from the cells. Total RNA was extracted using an RNA extraction kit (RNeasy mini; Qiagen, Tokyo, Japan) and was used to synthesize cDNA with reverse transcriptase (ReverTra Ace; Toyobo, Osaka, Japan). The sequences of the primers and conditions for PCR used are shown in Table 1. The PCR products were subjected to $2 \%$ agarose gel electrophoresis and stained with ethidium bromide.

Quantitative real-time PCR was performed according to a standard protocol using the SYBR Green PCR Master Mix (Toyobo) and ABI Prism 7700 Sequence Detection System (PE Applied Biosystems, Warrington, UK). Cycling conditions were incubation at $50^{\circ} \mathrm{C}$ for $2 \mathrm{~min}, 95^{\circ} \mathrm{C}$ for $10 \mathrm{~min}$, and 40 cycles of $95^{\circ} \mathrm{C}$ for $15 \mathrm{~s}$ and $60^{\circ} \mathrm{C}$ for $1 \mathrm{~min}$. Fold difference compared with glyceraldehyde-3-phosphate dehydrogenase expression was calculated.

\section{Western Blot Analysis}

Total proteins were extracted from the cells using T-PER protein extraction reagent (Pierce Chemical, Rockford, IL, USA). The protein was subjected to $10 \%$ SDS-polyacrylamide
Table 1 Sequences of the primers and PCR conditions used in this study

\begin{tabular}{llccc}
\hline Gene & Sequences $\left(5^{\prime}-3^{\prime}\right)$ & $\begin{array}{c}\text { Annealing } \\
\text { temperature } \\
\left({ }^{\circ} \mathrm{C}\right)\end{array}$ & $\begin{array}{c}\text { PCR } \\
\text { cycles }\end{array}$ & $\begin{array}{c}\text { Product } \\
\text { size } \\
(\mathrm{bp})\end{array}$ \\
\hline CK19 & $\begin{array}{l}\text { tcccgcgactacagccactactacacgacc } \\
\text { cgcgacttgatgtccatgagccgctggta }\end{array}$ & 60 & 30 & 745 \\
AFP & $\begin{array}{l}\text { gggagcggctgacattatta } \\
\text { tcttgcttcatcgtttgcag }\end{array}$ & 55 & 40 & 231 \\
Vimentin & $\begin{array}{l}\text { ctcggtggacttctcgctggcc } \\
\text { tcctgcaggttcttggcagcc }\end{array}$ & 67 & 25 & 612 \\
EGFR & $\begin{array}{l}\text { cctttggggcatagatcaga } \\
\text { gctgacctggagggaacata }\end{array}$ & 55 & 40 & 172 \\
GAPDH & $\begin{array}{l}\text { gagtcaacggatttggtcgt } \\
\text { ttgattttggagggatctc }\end{array}$ & 55 & 30 & 240 \\
CK19 & ggtcagtgtggaggtggatt & 60 & 40 & 198 \\
Real-time & tcagtaacctcggacctgct & & & \\
PCR & & & & \\
\hline
\end{tabular}

Abbreviations: AFP, $\alpha$-fetoprotein; CK, cytokeratin; EGFR, epidermal growth factor receptor; GAPDH, glyceraldehyde-3-phosphate dehydrogenase.

electrophoresis, and then electrophoretically transferred on to a nitrocellulose membrane. The membrane was incubated with primary antibodies against CK19 (1:100, RCK108, mouse monoclonal; DakoCytomation, Glostrup, Denmark), $\alpha$-fetoprotein (AFP; 1:500, rabbit polyclonal; DakoCytomation), EGF receptor (EGFR; 1:100, rabbit polyclonal; Santa Cruz Biotechnology, Santa Cruz, CA, USA), phospho-JNK/ stress-activated protein kinase (SAPK; 1:1000, rabbit monoclonal), phospho-p38 mitogen-activated protein kinase (MAPK; 1:1000, rabbit monoclonal), phospho-p44/42 MAPK (extracellular signal-regulated kinase1/2, ERK1/2; 1:2000, rabbit monoclonal), phospho-Akt (1:2000, rabbit monoclonal; all from Cell Signaling Technology, Danvers, MA, USA), and actin (1:3000, AC-15, mouse monoclonal; Abcam, Cambridge, MA, USA). The protein expression was detected using an EnVision + system (DakoCytomation), and 3,3'diaminobenzidine tetrahydrochloride (DAB) was used as the chromogen. Semiquantitative analysis of the results was performed using NIH J image software (National institutes of Health, Bethesda, MD, UAS). The fold difference compared with actin expression was calculated.

\section{Immunofluorescence Staining}

Cultured cells grown in a Lab-Tek chamber (Nalge Nunc International, Naperville, IL, USA) were assessed. Treatment with EGF $(30 \mathrm{ng} / \mathrm{ml}$ ) was performed for 5 days. The cells were fixed with $4 \%$ paraformaldehyde for $15 \mathrm{~min}$ and permeabilized for $3 \mathrm{~min}$ with $0.1 \%$ Triton X-100. After blocking, the cells were incubated overnight at $4{ }^{\circ} \mathrm{C}$ with primary 
antibodies against CK19 (1:100, DakoCytomation), and AFP (1:500, DakoCytomation). Color development was performed using the Vector Red alkaline phosphatase substrate kit (Vector Laboratories, Burlingame, CA, USA). Nuclei were stained with 4'6-diamino-2-phenylindole. The sections were observed under a fluorescence microscope. Negative controls were carried out by substitution of the primary antibodies with non-immunized serum.

\section{WST1 Cell Proliferation Assay}

The effects of EGF on cell proliferative activity were assessed using a WST1 assay (Roche Diagnostics KK, Indianapolis, IN, USA). The cells were seeded on 96 well dishes, and were incubated with standard medium for $24 \mathrm{~h}$. After the preincubation, the medium was exchanged for that containing EGF $(30 \mathrm{ng} / \mathrm{ml})$, and the cells were further incubated at indicated intervals. Then, the WST1 reagent was added, and the cell proliferative activity was determined spectrometrically. Each experiment was conducted in five sets.

\section{Invasion Assay}

Invasion assays were performed using Bio-coat cell migration chambers (BD Biosciences, Bedford, MA, USA) according to the manufacturer's instructions. A total of $5 \times 10^{4}$ cells were seeded on cell culture inserts, and the effects of EGF $(30 \mathrm{ng} / \mathrm{ml})$ on the invasive properties was determined. After $72 \mathrm{~h}$ after EGF stimulation, the cells were fixed in $100 \%$ methanol, and stained with hematoxylin. Cells that had migrated to the bottom side of the membrane were visualized under the microscope, and quantified by counting the number of cells in six randomly chosen visual fields.

\section{Liver Specimens}

A total of 78 patients with HCC, in which curative surgical hepatectomy was performed, were surveyed. The diagnosis of HCC was confirmed by the clinical and radiological data, and the histology of resected specimens. In this study, combined hepatocellular cholangiocarcinoma was not included. Such cases as relapse, non-curative resection, having history of anticancer treatment before operation, having cancerous thrombi in the portal vein, and having extrahepatic metastasis at the time of operation were excluded for the analysis. Tumor staging was defined according to the sixth edition of tumor-node-metastasis classification of Unio Internationale Contra Cancrum. The follow-up period ranged from 6 to 60 months. The diagnosis of recurrence was made on the basis of typical imaging appearance. Patients who had recurrent tumor received further treatments such as hepatectomy, radiofrequency ablation, transcatheter arterial chemoembolization, or chemotherapy, in which the adaptation was determined based on each patient condition.

\section{Immunohistochemistry}

Immunostaining was performed using formalin-fixed, paraffin-embedded tissue sections of surgically resected liver specimens of 78 HCC patients. After blocking the endogenous peroxidase, the sections were incubated in protein block solution (DakoCytomation). For the immunostaining of CK19 and JNK, antigen retrieval was performed by microwaving in $10 \mathrm{mmol} / \mathrm{l}$ citrate buffer $\mathrm{pH}$ 6.0. The sections were incubated overnight at $4{ }^{\circ} \mathrm{C}$ with primary antibodies against CK19 (1:100, DakoCytomation), EGF (1:50, goat polyclonal; Santa Cruz Biotechnology), and JNK (1:50, mouse monoclonal; Santa Cruz Biotechnology). Then the sections were incubated with secondary antibodies conjugated to peroxidase-labeled polymer, EnVision system (DakoCytomation) or Histofine Simple Stain MAX PO(G) (Nichirei, Tokyo, Japan). Color development was performed using DAB, and the sections were counterstained with hematoxylin. Negative controls were carried out by substitution of the primary antibodies with non-immunized serum, resulted in no signal detection.

Immunostaining of EGFR was performed using the commercially available kit, EGF pharmDx (DakoCytomation) according to manufacturer's instructions.

Double immunostaining of EGFR and CK19 was performed for the liver sections. First, immunostaining of EGFR was performed as above. After microwaving in $10 \mathrm{mmol} / \mathrm{l}$ citrate buffer $\mathrm{pH} 6.0$ for $10 \mathrm{~min}$, the sections were then incubated overnight at $4^{\circ} \mathrm{C}$ with the anti-CK19 antibody (1:100, DakoCytomation.). Color development was performed using the Vector Red alkaline phosphatase substrate kit (Vector Laboratories), and the sections were counterstained with hematoxylin.

Semiquantitative analysis of the results of immunostaining was performed for EGF, EGFR, and JNK. The results of immunostaining were categorized as follows: $1+, 1-10 \%$ positive; $2+, 11-50 \%$ positive; $3+, 51-100 \%$ positive.

\section{In Situ Hybridization}

Single-strand RNA probes for the EGFR were obtained by RT-PCR and in vitro transcription. Briefly, the T7- or SP6-RNA polymerase promoter was attached to primer. Then, single strand RNA probes complementary (antisense) and anticomplementary (sense) to the corresponding gene transcripts were obtained using in vitro transcription according to the standard protocol of the digoxigenin RNA transcription kit (Roche Diagnostics, Basel, Switzerland). The paraffin sections were incubated with hybridization solution (DakoCytomation) mixed with the digoxigenin-labeled antisense or sense probes at $50^{\circ} \mathrm{C}$ for $20 \mathrm{~h}$. After washing, the sections were incubated with alkaline phosphataseconjugated antidigoxigenin antibody (Roche Diagnostics) at room temperature for $1 \mathrm{~h}$. Color development was performed with a nitroblue tetrazolium salt/5-bromo-4-chloro-3-indolyl phospate toluidine salt solution (Roche Diagnostics) for $1 \mathrm{~h}$.

\section{Statistics}

The data were expressed as the mean \pm s.d. Statistical significance was determined using the Mann-Whitney $U$-test. Cumulative survival time was calculated by the Kaplan-Meier 
method and analyzed by the log-rank test. The relationship among CK19 immunoreactivity and clinicopathological parameters was analyzed using Mann-Whitney $U$-test and Chi square test. A $P$-value $<0.05$ was accepted as the level of statistical significance.

\section{RESULTS}

\section{Expression Analysis Of CK19 mRNA after Growth Factor} Stimulation in HCC Cell Line

At first, the effect of growth factor stimulation on CK19 mRNA expression was examined using a HCC cell line, PLC-5. PLC-5 was treated with EGF, TGF- $\beta 1$, HGF, and FGF-b, and the expression of CK19 mRNA was examined using RT-PCR. Among the growth factors tested, EGF had a prominent effect on the induction of CK19 mRNA in PLC5, and the mRNA expression of AFP was reduced (Figure 1a). Treatment with HGF and FGF-b seemed to have little effects on the mRNA expression of CK19 and AFP, whereas TGF- $\beta 1$ reduced CK19 and AFP mRNA expression (Figure 1a). TGF- $\beta 1$ induced the mRNA expression of vimentin, a hall marker of mesenchymal cells, and the expression of vimentin mRNA was unchanged after stimulation with EGF, HGF, and FGF-b (Figure 1a).

On phase-contrast microscope, no morphological alteration was observed after treatment with EGF, HGF and FGF-b, whereas TGF- $\beta 1$ induced spindle cell morphology, simulating the phenomenon of epithelial-to-mesenchymal transition (Figure 1b). On the basis of these results, we focused on the effects of EGF, and further in vitro analysis was performed using two HCC cell lines (PLC-5, HepG2).

a
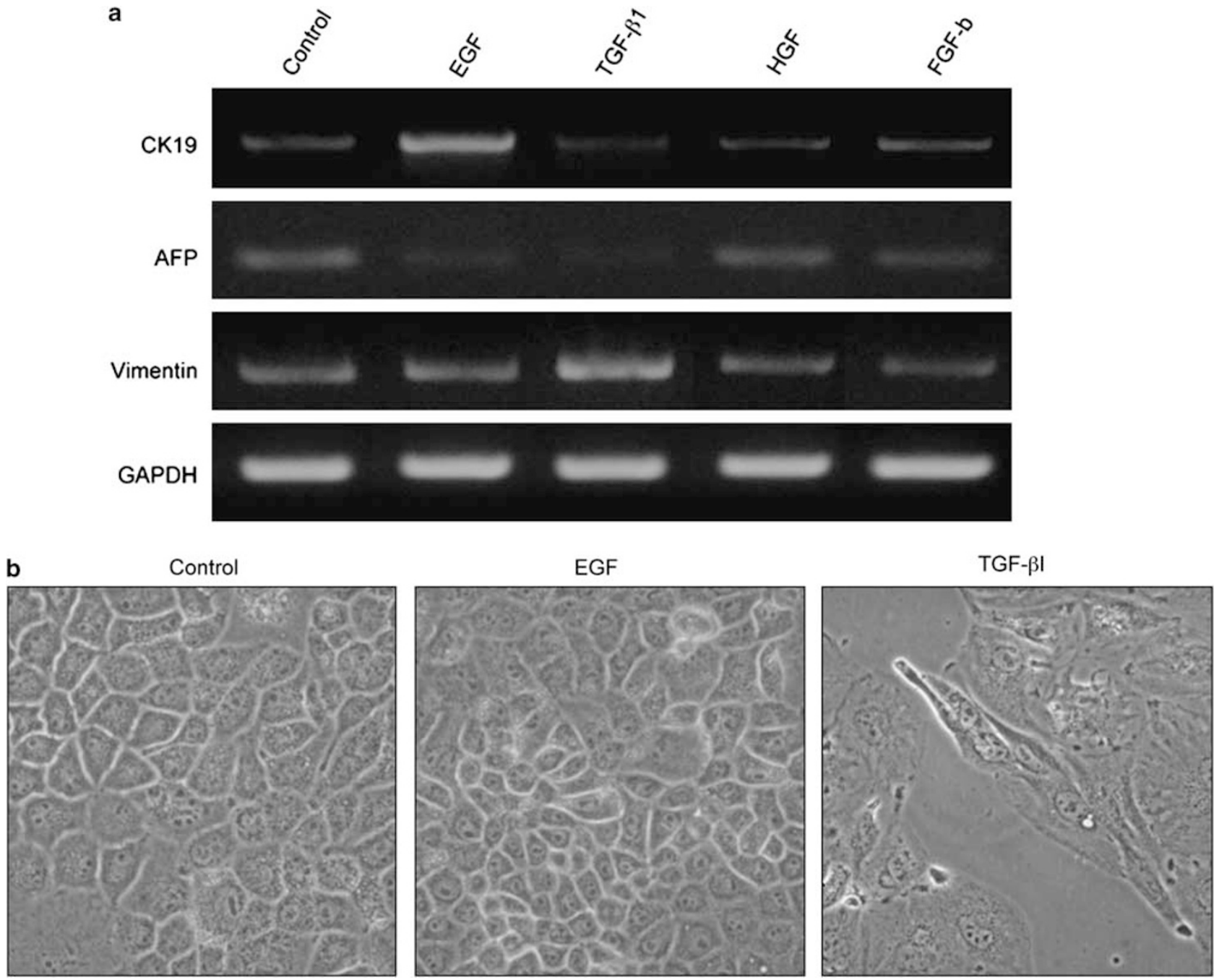

Figure 1 Expression analysis of CK19 mRNA after growth factor stimulation in HCC cell line. A human HCC cell line PLC-5 was treated with EGF, TGF- $\beta 1$, HGF and FGF-b, and the mRNA expression of CK19, AFP and vimentin was examined by RT-PCR (a). EGF had a prominent effect on the induction of CK19 mRNA, accompanied by the reduction of AFP mRNA. The mRNA expression of vimentin was induced by TGF- $\beta 1$ stimulation. On phase-contrast microscope, no morphological alteration was observed after treatment with EGF, whereas TGF- $\beta 1$ induced spindle cell morphology (b). Original magnifications, $\times 200$ (b). 

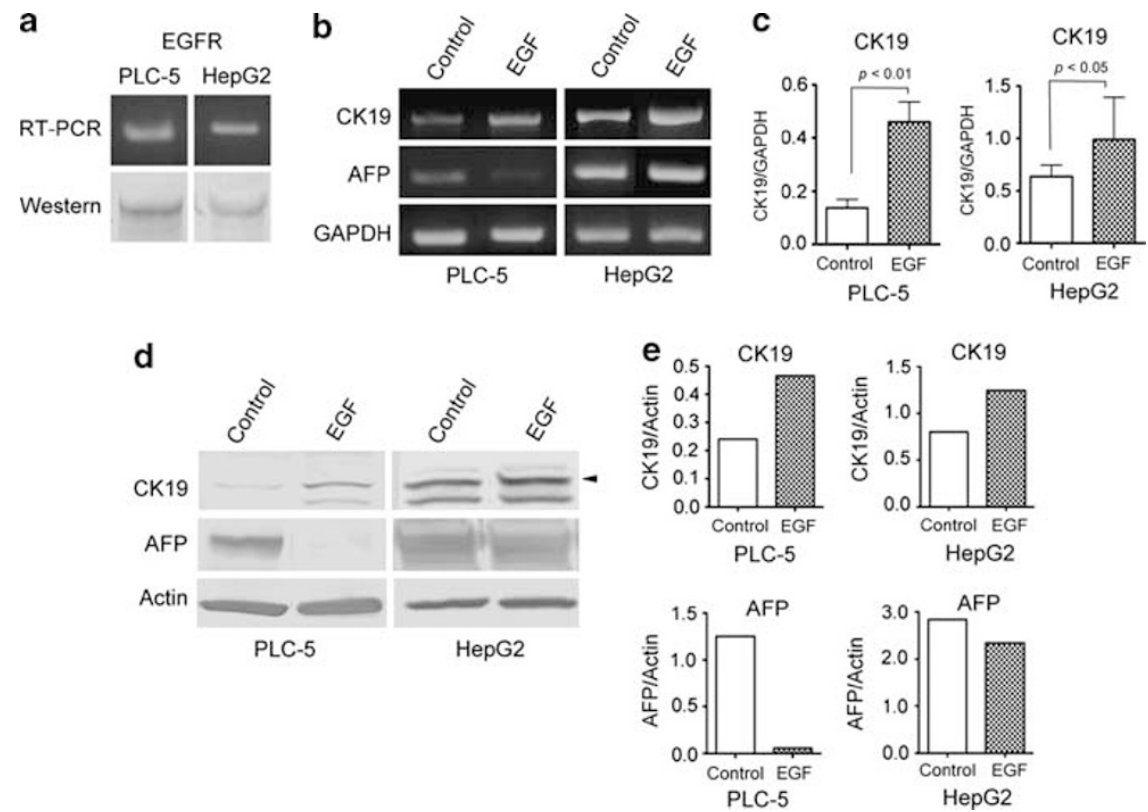

Figure 2 Induction of CK19 expression in HCC cell lines EGF stimulation. Two human HCC cell lines, PLC-5 and HepG2, expressed EGFR at the mRNA and protein levels (a). Stimulation of the cells with EGF induced CK19 mRNA expression, which was examined using RT-PCR (b) and real-time PCR (c). Western blot analysis showed the induction of CK19 protein in PLC-5 and HepG2 following EGF treatment (d), and semiquantitative analysis of the results confirmed this tendency (e). EGF reduced the expression of AFP in PLC-5 at the mRNA and protein levels, but their expression was unaffected in HepG2 (b, d, e). The data were expressed as the mean \pm s.d. of six (c), and the mean of two sets (e).

Induction Of CK19 Expression In HCC Cell Lines By EGF

RT-PCR and western blot analysis confirmed that both PLC-5 and HepG2 expressed EGFR at the mRNA and protein levels (Figure 2a). EGF induced CK19 mRNA expression as assessed by RT-PCR (Figure 2b), and the results of real-time PCR confirmed that EGF significantly induced CK19 mRNA expression in both cell lines (Figure 2c). Western blot analysis also showed the induction of the expression of CK19 protein after EGF treatment in both cell lines (Figures 2d and 2e). EGF dramatically reduced the expression of AFP in PLC-5 at the mRNA and protein levels, but its expression was unaffected in HepG2 (Figures 2b, d and e).

Immunofluorescence staining of CK19 and AFP demonstrated that PLC-5 diffusely expressed AFP in their cytoplasm, whereas smaller number of cells showed positive immunoreactivity of CK19 in the control (no EGF treatment) group (Figure 3). After EGF treatment, most of the cells were strongly positive for CK19, and the expression of AFP was markedly reduced (Figure 3), which were consistent with the results of real-time PCR and western blotting.

\section{Phosphorylation Of JNK/SAPK In HCC Cell Lines after EGF Stimulation}

To determine the intracellular signaling pathway involved in the induction of CK19 expression, the expression of the phosphorylated form of JNK/SAPK, p38 MAPK, ERK1/2 and Akt was examined using western blotting. In PLC-5 and HepG2, the levels of phospho-JNK/SAPK expression increased after EGF treatment (Figures 4a and b). By contrast, the expression of phospho-p38 MAPK, ERK1/2 and Akt was unaffected by the treatment in both cell lines (data not shown).

To further examine the involvement of JNK/SAPK in the induction of CK19 expression, PLC-5 was treated with JNK inhibitors (JNK inhibitor I, JNK inhibitor II) in combination with EGF, and the expression of CK19 was examined by western blottng. As shown in Figures $4 \mathrm{c}$ and d, both JNK inhibitors suppressed the EGF-meditated induction of CK19 expression, indicating that JNK/SAPK is a downstream regulator of CK19 expression via EGF-EGFR. In addition, the JNK inhibitors reversed the EGF-mediated reduction of AFP expression in PLC-5 (Figures 4c and d).

\section{Increase in Proliferative Ability and Invasive Property of HCC Cell Lines by EGF}

The WST1 cell proliferation assay showed that the proliferative ability of the cells was higher in the EGF-treated groups than that of the control group in both PLC-5 and HepG2 (Figure 5a). Invasive properties of PLC-5 and HepG2 also significantly increased after EGF treatment, which was determined using the invasion assay (Figure 5b). Representative images of the invasion assay are shown in Figure 5c.

\section{Expression Of CK19, EGF, EGFR, and JNK in Human HCC Tissues}

Positive immunoexpression of CK19 in human HCC tissues was observed in limited number of cases. In most of the 


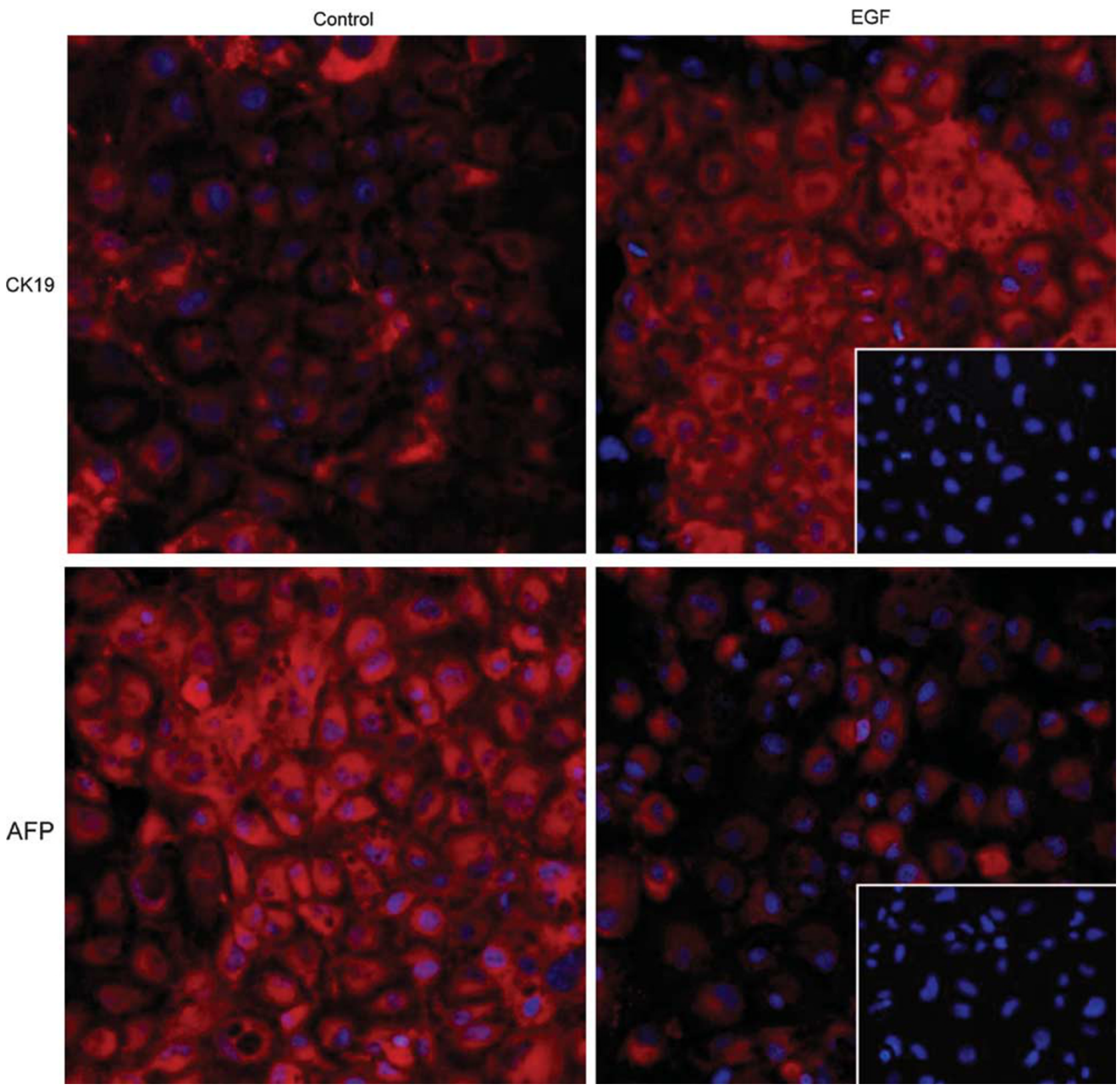

Figure 3 Alteration of CK19 and AFP expression in PLC-5 after EGF stimulation Immunofluorescence staining of CK19 and AFP was performed for PLC-5 with and without EGF treatment. CK19 and AFP were visualized by the Vector Red alkaline phosphatase substrate (red), and nuclei were stained with 4'6diamino-2-phenylindole (blue). EGF markedly induced CK19 expression in PLC-5, and the expression of AFP was reduced. Negative controls for CK19 and AFP immunofluorescence staining were shown in the insets. Original magnifications, $x 200$.

CK19-positive cases, CK19 expression in HCC was not diffusely observed, and CK19-positive cells were aggregated in focal areas in a single section (Figure 6b). On the basis of previous reports, ${ }^{8,11,12}$ CK19-positive HCC was defined as such tumor that $<5 \%$ of total carcinoma cells showed positive immunoreactivity of CK19 in this study. According to this definition, $9(12 \%)$ of 78 cases of surgically resected HCC samples corresponded to CK19-positive HCC.

Immunohistochemical expression of EGF and EGFR in HCC varied from case to case (Figures $6 \mathrm{c}$ and d). Semi- quantitative analysis of the results of immunostaining showed that the positive immunohistochemical signals of EGF and EGFR were significantly more frequent in CK19-positive HCC compared with those of CK19-negative HCC $(P<0.01$ and $P<0.05$, respectively; Figure 6j). It is of note that all cases of CK19-positive HCC $(n=9)$ showed positive immunohistochemical expression of EGF and EGFR (Figure 6j). Double immunostaining for CK19 and EGFR showed that EGFR was colocalized with CK19 (Figure 6e). In situ hybridization studies further 

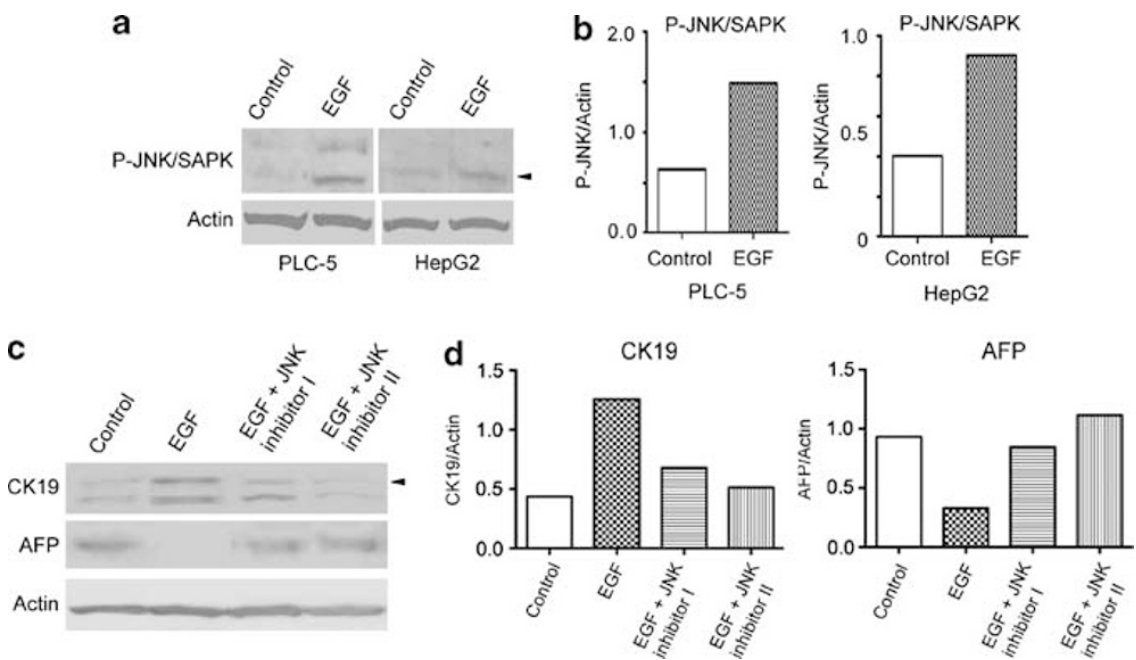

Figure 4 Phosphorylation of JNK/SAPK in HCC cell lines after EGF stimulation. Western blot analysis showed that the expression of phosphor-JNK/SAPK was increased in PLC-5 and HepG2 after EGF treatment (a). JNK inhibitor I and JNK inhibitor II blocked EGF-induced CK19 expression and EGF-induced AFP reduction in PLC-5 (c). The results of semiquantitative analysis of the western blotting were shown in (b) and (d). The data were expressed as the mean of two sets $(\mathbf{b}, \mathbf{d})$.
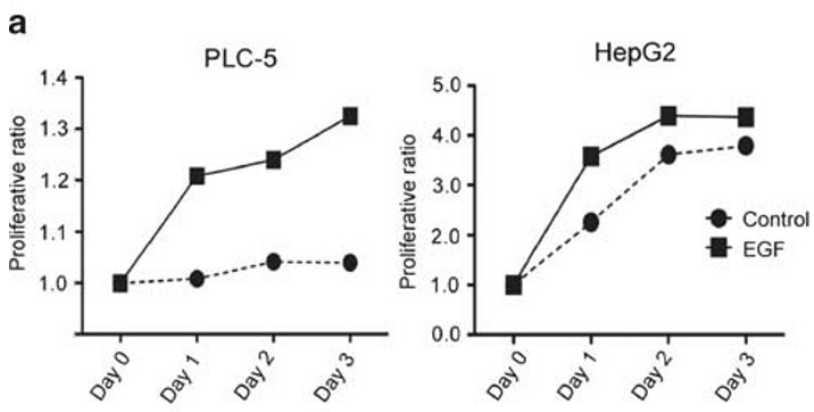

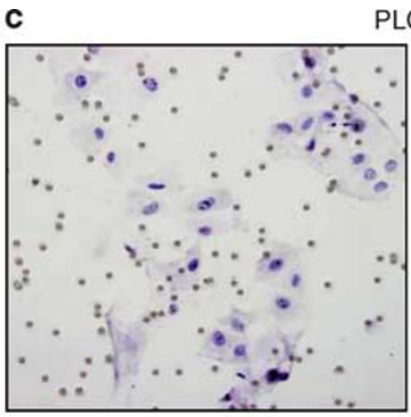

Control
PLC-5

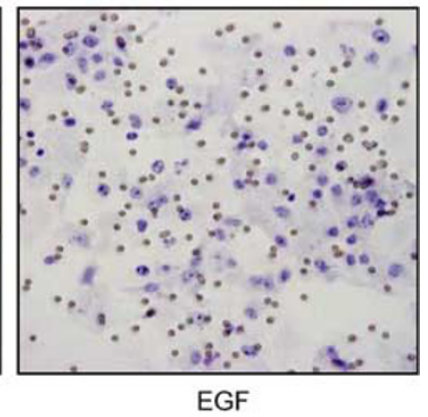

b

PLC-5
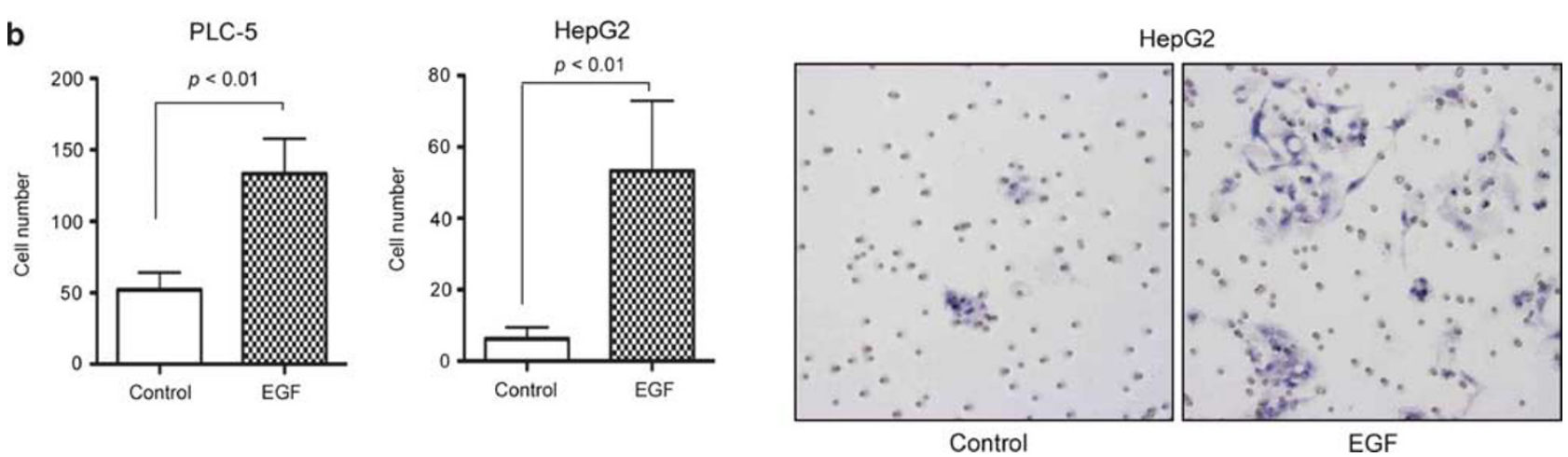

Figure 5 Increase in proliferative ability and invasive property of HCC cell lines by EGF. PLC-5 and HepG2 were treated with EGF, and the cell proliferative ability and invasive property were examined using the WST1 cell proliferation assay and the invasion assay, respectively. The cell proliferative ability was higher in EGF-treated group that that of control group in both PLC-5 and HepG2 (a). Invasive properties of PLC-5 and HepG2 was significantly increased after EGF treatment (b). Representative images of the invasion assay were shown in (c). Hematoxylin-stained blue nuclei indicate migrated cells (c). The data were expressed as the mean \pm s.d. of five (a) and six sets (b). Original magnifications, $\times 200$ (c).

confirmed that CK19-positive HCC expressed EGFR mRNA (Figure $6 \mathrm{f}$ and $\mathrm{g}$ ).

All cases of CK19-positve HCC showed immunohistochemical expression of JNK. Although the signal intensity of JNK was observed heterogeneously in a single section, it tended to be preferably expressed in the CK19-positive areas (Figure 6h and i; the lower part of the figures was CK19-positive area, and the upper part was CK-19-negative area). Semiquantitative analysis of the immunohistochemical expression of JNK showed that its expression was 

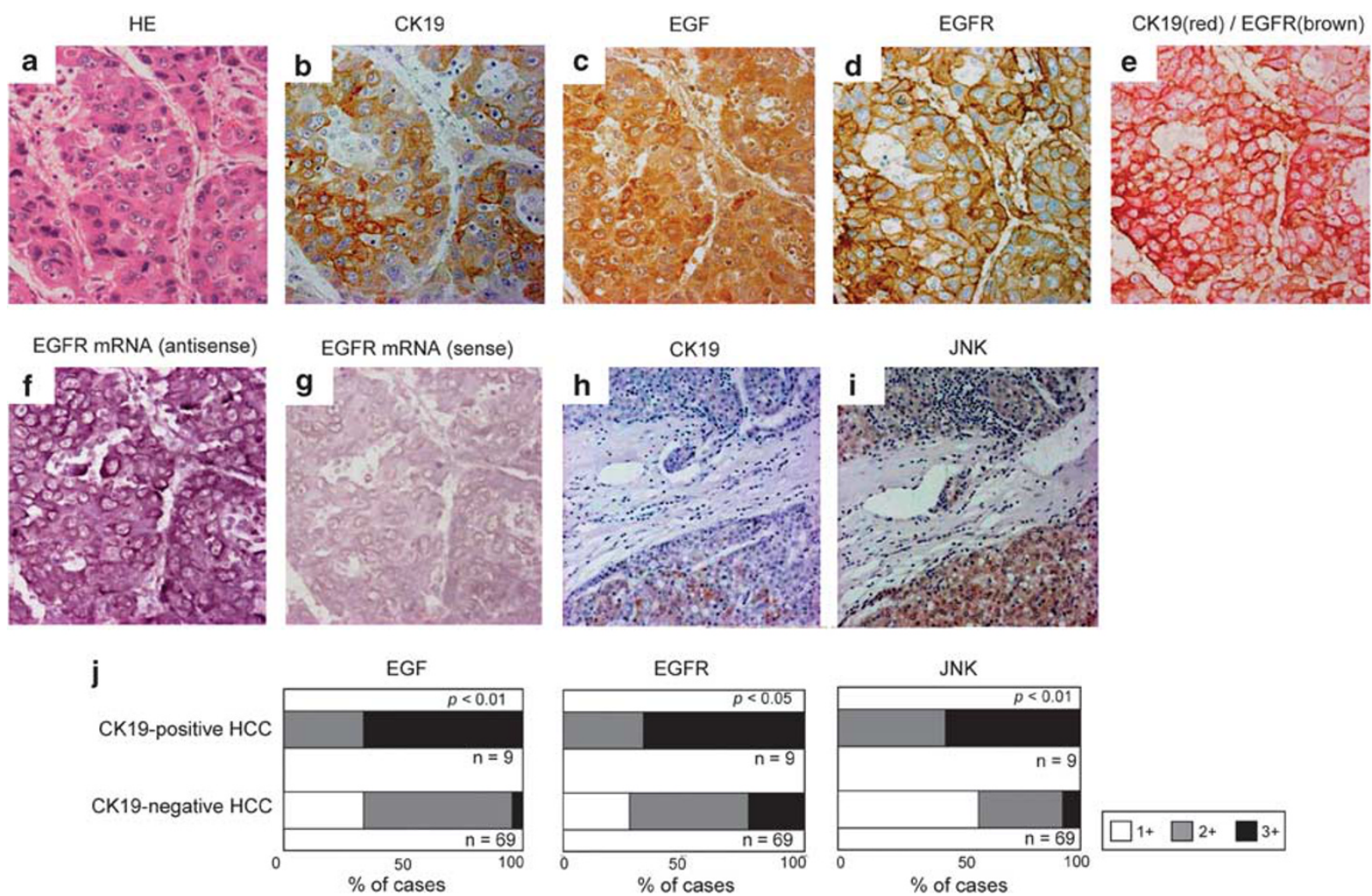

Figure 6 Expression of CK19, EGF, EGFR, and JNK in human HCC tissues. Examples of the results of histological analysis for one CK19-positive HCC case are shown (a-i). Carcinoma cells expressed CK19 (b), EGF (c) and EGFR (d). CK19 expression tended to be focal, and the expression of EGF and EGFR was diffuse in a single section. Double immunostaining of EGFR (brown) and CK19 (red) showed their colocalization (e). In situ hybridization studies confirmed that CK19-positive HCC expressed EGFR mRNA (f, $\mathbf{g})$. The immunohistochemical expression of JNK was preferably observed in the CK19-positive areas (h, $\mathbf{i}$; the lower part of the figures was CK19-positive area, and the upper part was CK-19-negative area). Semiquantitative analysis of the results of EGF, EGFR, and JNK immunostaining was performed as described in the Materials and Methods. The analysis showed that the extent of positive immunohistochemical signals of EGFR, EGF and JNK was significantly intense in CK19-positive HCC (j). Original magnifications; x400 (a-g), x100 (h, i).

significantly more frequent in CK19-positive HCC compared with that of CK19-negative HCC $(P<0.01$; Figure 6j).

\section{Poor Prognosis of CK19-Positive HCC Patients}

The results of clinicopathological analysis are summarized in Table 2. Portal vein invasion was observed more frequently in CK19-positive HCC compared with that of CK19-negative HCC, and there was a significant difference between them $(P=0.0348)$. In addition, occurrence of extrahepatic metastasis after curative surgical hepatectomy was observed more frequently in CK19-positive HCC during the follow up period $(P=0.0085)$. Extrahepatic metastasis was observed in three patients (43\%) of CK19-positive HCC, in which two patients had bone metastasis, and one had adrenal metastasis. Other clinicopathlogical factors listed in Table 2 did not correlate significantly with CK19 expression in HCC.

In the whole study population, the 5-year disease free survival (DFS) and overall survival (OS) rates were 25.4 and $85.4 \%$, respectively. CK19 positivity significantly correlated with the 2-year DFS rate $(P=0.0171$; Figure $7 \mathrm{a})$. Both 5-year DFS and OS rates tended to be lower in CK19-positive HCC group, but CK19 positivity did not significantly correlated with the 5-year DFS rate $(P=0.0894)$ and the 5-year OS rate $(P=0.6272$; Figures $7 \mathrm{~b}$ and $\mathrm{c})$.

\section{DISCUSSION}

This study demonstrated that EGF induced CK19 expression in well-established HCC cell lines, PLC-5 and HepG2, and revealed a possible mechanism underlying the development of CK19-positive HCC. In PLC-5, the induction of CK19 expression was accompanied by the marked reduction of AFP expression, indicating phenotypic transdifferentiation of the cells from hepatocellular-to-biliary phenotype.

In rat mature hepatocyte, transdifferentiation of hepatocyte to biliary epithelium has been demonstrated in experimental rodent models, hepatocyte organoid culture, and three-dimensional hepatocyte culture. ${ }^{18-22}$ In these studies, EGF has been shown to be an essential promoter of the phenotypic transdifferentiation of mature hepatocytes. ${ }^{18,20-22}$ This study demonstrated that EGF could induce phenotypic changes in HCC similar to those seen in non-cancerous mature hepatocytes. Previous studies also showed that HGF was another promoter of the phenotypic changes of mature hepatocytes. ${ }^{18,21,22}$ However, as shown in Figure 1a, our 
Table 2 Correlation between CK19 expression and clinicopathological factors in patients with hepatocellular carcinoma

\begin{tabular}{lccc}
\hline & CK19 negative & CK19 positive & $P$-value \\
\hline Patients & 69 & 9 & \\
Sex & & & \\
$\quad$ Male & 57 & 7 & 0.7224 \\
$\quad$ Female & 12 & 2 & \\
Age (mean) & 67 & 60 & 0.1308 \\
& & & \\
Virus & & & \\
$\quad$ HBV & 7 & 1 & 0.9284 \\
HCV & 51 & 8 & 0.3249 \\
Non-B non-C & 11 & 0 & 0.1962
\end{tabular}

\section{Preoperative AFP}

$>20$

$<20$

\section{Child-pugh score}

A

B

\section{TNM stage}

I-II

III-IV

Portal vein invasion

Positive

Negative

Tumor size

$$
>5 \mathrm{~cm}
$$$$
<5 \mathrm{~cm}
$$

Tumor number

Multiple

Single

Encapsulation

Positive

Negative

Histology

Well

Mod

Por
0.3249

0.1962

0.1729

0.8168

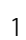

8

0.7103

preliminary studies failed to demonstrate significant changes in CK19 and AFP expression after HGF treatment in PLC-5.

The results of this study indicated that CK19 expression in HCC might be an acquired feature of the carcinoma cells once conventional HCC had arisen. Most carcinomas show phenotypic heterogeneity, ${ }^{27}$ and CK19 expression in HCC is one of such examples. The heterogeneity may result from tumor cell renewal and adaptation to specific microenvironment. The presence of cancer stem cells may be another contributor of the heterogeneity. ${ }^{28,29}$ The results obtained here may also be implicated in the histogenesis of combined hepatocellular cholangiocarcinoma; that is, the component of cholangiocarcinoma originates from the transdifferention of the HCC component, forming a combined hepatocellular cholangiocarcinoma that exhibits focal or diffuse immunohistochemical expression of CK19. ${ }^{30,31}$

The expression of EGFR has been observed in 40 to $70 \%$ of conventional HCC in previous studies. ${ }^{32-35} \mathrm{~A}$ recent study showed that the expression of EGF was upregulated in advanced HCC compared with that of control liver tissue and early HCC. $^{36}$ In this study, all cases of human CK19-positive HCC were found to express EGFR and EGF, and double immunostainig showed that CK19 was colocalized with EGFR. These findings indicate that carcinoma cells produce EGF, and it acts with EGFR in an autocrine and/or paracrine manner, which in turn induces CK19 expression and thus forming CK19-positive HCC.

In this study, the expression of CK19 was seen in relatively limited number of cases of HCC, and not all cases that showed immunohistochemical expression of EGFR and EGF were CK19-positive HCC. As shown in Figure 4, in vitro studies indicated that the JNK/SAPK signaling pathway might have an important role in the CK19 induction of HCC. In vivo, the extent of the positive immunohistochemical expression of JNK tended to be more intense in the CK19positive areas of HCC, and the JNK expression was observed more frequently in CK19-positive HCC. Therefore, the status of the expression of JNK may be one of the factors which account for the facts that all EGFR- and EGF-positive HCC did not express CK19. In addition, two HCC cell lines exhibited different responses to EGF in vitro; that is, the 

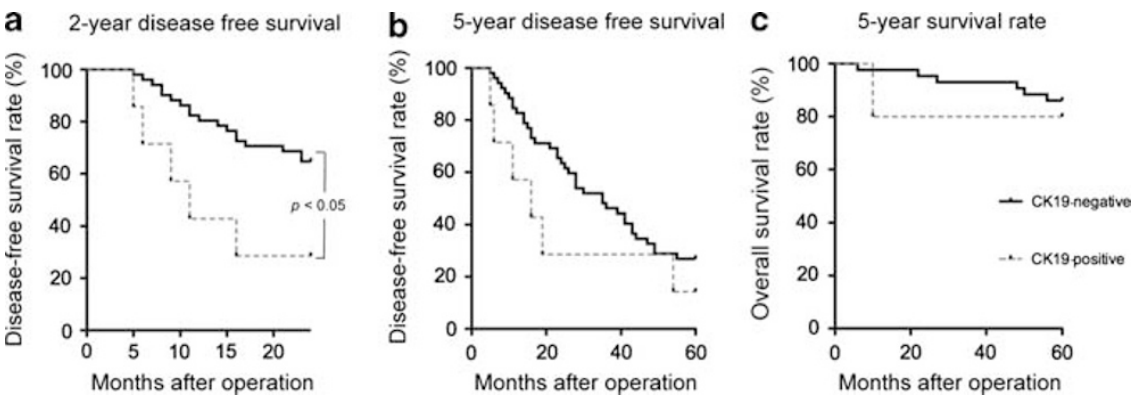

Figure 7 Poor prognosis of CK19-positive HCC patients. The correlation with the CK19 immunoreactivity and the DFS and OS rates were examined for 78 HCC patients. CK19 positivity significantly correlated with the 2-year DFS rate $(P=0.0171)(\mathbf{a})$. Both 5 -year DFS rate (b) and OS rate (c) tended to be lower in CK19-positive HCC group, but CK19 positivity did not significantly correlated with the 5 -year DFS rate $(P=0.0894)$ and the 5 -year OS rate $(P=0.6272)$.

reduction of AFP expression occurred in PLC-5, whereas it did not reduced in HepG2, indicating complex intracellular signaling pathways involved in the EGF-EGFR axis in HCC.

In addition to the induction of CK19 expression, EGF increased proliferative abilities and invasive properties in PLC-5 and HepG2, demonstrating that stimulation with EGF led to more aggressive growth of HCC and increased malignant potential. These results were consistent with the findings of this study that CK19-positive HCC had a high incidence of portal vein invasion, extrahepatic metastasis, and an early relapse, which related to the worsened 2-year DFS rate.

The frequency of CK19-positive HCC was 12\% (9/78 cases) in this study, and the frequency was almost consistent with previously reported data on the frequency of CK19positive HCC (10-19\%). ${ }^{8,11,12}$ In accordance with these reports, CK19 positivity significantly correlated with the 2-year DFS rate. ${ }^{811}$ Although the 5 -year DFS and OS rate was not significantly correlated with CK19 positivity in this study, both of them tended to be lower in CK19-positive HCC group. Because this study dealt with relatively limited number of cases and the follow up periods were not sufficient, a significant difference might not be obtained.

In summary, this study elucidated an underlying mechanism of the development of CK19-positive HCC. The activation of the EGF-EGFR signaling pathway via phosphorylation of JNK/ SAPK may be closely associated with the histogenesis of CK19positive HCC. EGF increased the abilities of cell growth and invasive properties of HCC cell lines, showing an acquisition of more malignant potential and accounting for the poor prognosis of the patients. This study also confirmed previously reported findings that CK19-positive HCC had a poor prognosis. These results suggest that the inhibition of EGF-EGFR axis may improve the poor prognosis of the patients of CK19positive HCC, implying as a potential therapeutic target.

\section{DISCLOSURE/CONFLICT OF INTEREST}

The authors declare no conflict of interest.

1. Parkin D. Global cancer statistics in the year 2000. Lancet Oncol 2001;2:533-543.

2. Jemal A, Siegel R, Ward E, et al. Cancer statistics. CA Cancer J Clin 2007;57:43-66.
3. Tang Z, Ye S, Liu Y, et al. A decade's studies on metastasis of hepatocellular carcinoma. J Cancer Res Clin Oncol 2004;130:187-196.

4. Yang $\mathrm{Y}$, Nagano $\mathrm{H}$, Ota $\mathrm{H}$, et al. Patterns and clinicopathologic features of extrahepatic recurrence of hepatocellular carcinoma after curative resection. Surgery 2007;141:196-202.

5. Van Eyken P, Sciot R, Paterson A, et al. Cytokeratin expression in hepatocellular carcinoma: an immunohistochemical study. Hum Pathol 1988; 19:562-568.

6. Robrechts C, De Vos R, Van den Heuvel M, et al. Primary liver tumour of intermediate (hepatocyte-bile duct cell) phenotype: a progenitor cell tumour? Liver 1998;18:288-293.

7. Yoon $\mathrm{D}$, Jeong J, Park $\mathrm{Y}$, et al. Expression of biliary antigen and its clinical significance in hepatocellular carcinoma. Yonsei Med J 1999;40:472-477.

8. Uenishi T, Kubo S, Yamamoto T, et al. Cytokeratin 19 expression in hepatocellular carcinoma predicts early postoperative recurrence. Cancer Sci 2003;94:851-857.

9. Wu P, Fang J, Lau V, et al. Classification of hepatocellular carcinoma according to hepatocellular and biliary differentiation markers. Clinical and biological implications. Am J Pathol 1996;149:1167-1175.

10. Ding $\mathrm{S}, \mathrm{Li} \mathrm{Y}, \operatorname{Tan} \mathrm{Y}$, et al. From proteomic analysis to clinical significance: overexpression of cytokeratin 19 correlates with hepatocellular carcinoma metastasis. Mol Cell Proteomics 2004;3:73-81.

11. Yang X, Xu Y, Shi G, et al. Cytokeratin 10 and cytokeratin 19: predictive markers for poor prognosis in hepatocellular carcinoma patients after curative resection. Clin Cancer Res 2008;14:3850-3859.

12. Durnez A, Verslype C, Nevens F, et al. The clinicopathological and prognostic relevance of cytokeratin 7 and 19 expression in hepatocellular carcinoma. A possible progenitor cell origin. Histopathology 2006:49:138-151.

13. Moll R, Franke W, Schiller $D$, et al. The catalog of human cytokeratins: patterns of expression in normal epithelia, tumors and cultured cells. Cell 1982;31:11-24.

14. Osborn $M$, van Lessen $G$, Weber $K$, et al. Differential diagnosis of gastrointestinal carcinomas by using monoclonal antibodies specific for individual keratin polypeptides. Lab Invest 1986;55:497-504.

15. van Eyken $P$, Sciot $R$, van Damme $B$, et al. Keratin immunohistochemistry in normal human liver. Cytokeratin pattern of hepatocytes, bile ducts and acinar gradient. Virchows Arch A Pathol Anat Histopathol 1987;412:63-72.

16. Lai $Y$, Thung $S$, Gerber $M$, et al. Expression of cytokeratins in normal and diseased livers and in primary liver carcinomas. Arch Pathol Lab Med 1989;113:134-138.

17. Roskams T, De Vos R, Van Eyken P, et al. Hepatic OV- 6 expression in human liver disease and rat experiments: evidence for hepatic progenitor cells in man. J Hepatol 1998;29:455-463.

18. Limaye $\mathrm{P}$, Bowen W, Orr A, et al. Mechanisms of hepatocyte growth factor-mediated and epidermal growth factor-mediated signaling in transdifferentiation of rat hepatocytes to biliary epithelium. Hepatology 2008;47:1702-1713.

19. Michalopoulos G, Barua L, Bowen W. Transdifferentiation of rat hepatocytes into biliary cells after bile duct ligation and toxic biliary injury. Hepatology 2005;41:535-544.

20. Nishikawa $Y$, Doi $Y$, Watanabe $H$, et al. Transdifferentiation of mature rat hepatocytes into bile duct-like cells in vitro. Am J Pathol 2005;166: 1077-1088. 
21. Michalopoulos G, Bowen W, Mulé K, et al. Hepatocytes undergo phenotypic transformation to biliary epithelium in organoid cultures Hepatology 2002;36:278-283.

22. Michalopoulos G, Bowen W, Mulé K, et al. Histological organization in hepatocyte organoid cultures. Am J Pathol 2001;159:1877-1887.

23. Del Castillo G, Alvarez-Barrientos A, Carmona-Cuenca I, et al. Isolation and characterization of a putative liver progenitor population after treatment of fetal pat hepatocytes with TGF-beta. J Cell Physiol 2008;215:846-855.

24. Yoshida M, Nishikawa $Y$, Omori $Y$, et al. Involvement of signaling of VEGF and TGF-beta in differentiation of sinusoidal endothelial cells during culture of fetal rat liver cells. Cell Tissue Res 2007;329: 273-282.

25. Xu C, Rosler E, Jiang J, et al. Basic fibroblast growth factor supports undifferentiated human embryonic stem cell growth without conditioned medium. Stem Cells 2005;23:315-323.

26. Zaret K. Hepatocyte differentiation: from the endoderm and beyond. Curr Opin Genet Dev 2001;11:568-574.

27. Heppner G. Tumor heterogeneity. Cancer Res 1984;44:2259-2265.

28. Vermeulen $L$, Sprick $M$, Kemper $K$, et al. Cancer stem cells-old concepts, new insights. Cell Death Differ 2008;15:947-958.

29. Bioulac-Sage P, Balabaud C, Wanless I. Diagnosis of focal nodular hyperplasia: not so easy. Am J Surg Pathol 2001;25:1322-1325.
30. Zhang F, Chen X, Zhang W, et al. Combined hepatocellular cholangiocarcinoma originating from hepatic progenitor cells: immunohistochemical and double-fluorescence immunostaining evidence. Histopathology 2008;52:224-232.

31. Aishima S, Nishihara Y, Kuroda Y, et al. Histologic characteristics and prognostic significance in small hepatocellular carcinoma with biliary differentiation: subdivision and comparison with ordinary hepatocellular carcinoma. Am J Surg Pathol 2007;31:783-791.

32. Buckley A, Burgart L, Sahai V, et al. Epidermal growth factor receptor expression and gene copy number in conventional hepatocellular carcinoma. Am J Clin Pathol 2008;129:245-251.

33. Tang Z, Qin L, Wang X, et al. Alterations of oncogenes, tumor suppressor genes and growth factors in hepatocellular carcinoma: with relation to tumor size and invasiveness. Chin Med J (Engl) 1998;111:313-318.

34. El-Bassiouni $A$, Nosseir $M$, Zoheiry $M$, et al. Immunohistochemical expression of CD95 (Fas), c-myc and epidermal growth factor receptor in hepatitis $C$ virus infection, cirrhotic liver disease and hepatocellular carcinoma. APMIS 2006;114:420-427.

35. Ito $Y$, Takeda T, Sasaki $Y$, et al. Expression and clinical significance of the erbB family in intrahepatic cholangiocellular carcinoma. Pathol Res Pract 2001;197:95-100.

36. Villanueva A, Chiang D, Newell $P$, et al. Pivotal role of mTOR signaling in hepatocellular carcinoma. Gastroenterology 2008;135:1972-1983. 